\title{
Expectation Identities of Generalized Order Statistics from Marshall-Olkin Extended Uniform Distribution and its Characterization
}

\author{
Haseeb Athar* and Nayabuddin \\ Department of Statistics \& Operations Research \\ Aligarh Muslim University, Aligarh - 202002, India \\ haseebathar@hotmail.com \\ Received 21 November 2013 \\ Accepted 30 December 2014
}

\begin{abstract}
The Marshall-Olkin extended uniform distribution is introduced and studied by Jose and Krishna (2011). In this paper some moments properties of generalized order statistics (gos) for this distribution are investigated and results are deduced for order statistics and record values. In the last section, a characterization result is presented.
\end{abstract}

Keywords: Marshall-Olkin extended uniform distribution; generalized order statistics; order statistics; record values; recurrence relations and characterization.

2000 Mathematics Subject Classification: 62G30, 62E10

\section{Introduction}

Kamps (1995) introduced the unifying concept of generalized order statistics (gos), the use of such concept has been steadily growing along the years. This is due to the fact that such concept describes random variables arranged in ascending order of magnitude and includes important well known concept that have been separately treated in statistical literature. Examples of such concepts are the order statistics, sequential order statistics, progressive type II censored order statistics, record values and Pfeifer's records. Application is multifarious in a variety of disciplines and particularly in reliability.

Let $n \geq 2$ be a given integer and $\tilde{m}=\left(m_{1}, m_{2}, \ldots, m_{n-1}\right) \in \Re^{n-1}, k \geq 1$ be the parameters such that

$$
\gamma_{i}=k+n-i+\sum_{j=i}^{n-1} m_{j} \geq 0 \text { for } 1 \leq i \leq n-1
$$

The random variables $X(1, n, \tilde{m}, k), X(2, n, \tilde{m}, k), \ldots, X(n, n, \tilde{m}, k)$ are said to be generalized order statistics from an absolutely continuous distribution function $F()$ with the probability density funtion $(p d f) f()$, if their joint density function is of the form

$\overline{{ }^{*} \text { Corresponding author }}$ 


$$
k\left(\prod_{j=1}^{n-1} \gamma_{j}\right)\left(\prod_{i=1}^{n-1}\left[1-F\left(x_{i}\right)\right]^{m_{i}} f\left(x_{i}\right)\right)\left[1-F\left(x_{n}\right)\right]^{k-1} f\left(x_{n}\right)
$$

on the cone $F^{-1}(0)<x_{1} \leq x_{2} \leq \ldots \leq x_{n}<F^{-1}(1)$.

If $m_{i}=0(i=1,2, \ldots, n-1), k=1$, we obtain the joint $p d f$ of the order statistics and for $m_{i}=$ $-1, k \in N$, we get $k-t h$ record values.

A random variable $X$ is said to have the Marshall-Olkin extended uniform distribution if its $p d f$ is of the form

$$
f(x)=\frac{\alpha \theta}{[\alpha \theta+(1-\alpha) x]^{2}}, \quad 0<x<\theta, \alpha>0
$$

and corresponding $d f$

$$
F(x)=\frac{x}{\alpha \theta+(1-\alpha) x}, \quad 0<x<\theta, \alpha>0
$$

Now in view of (1.2) and (1.3), we have

$$
\theta \bar{F}(x)=(\theta-x)[\alpha \theta+(1-\alpha) x] f(x)
$$

where, $\bar{F}(x)=1-F(x)$.

The relation (1.4) will be utilized to establish recurrence relations for moments of gos.

\section{Single Moments}

Case I: $\gamma_{i} \neq \gamma_{j} ; i, j=1,2, \ldots, n-1$.

In view of (1.1) the $p d f$ of $r-t h$ generalized order statistic $X(r, n, \tilde{m}, k)$ is

$$
f_{X(r, n, \tilde{m}, k)}(x)=C_{r-1} f(x) \sum_{i=1}^{r} a_{i}(r)[\bar{F}(x)]^{\gamma_{i}-1}
$$

where,

$$
C_{r-1}=\prod_{i=1}^{r} \gamma_{i}, \quad \gamma_{i}=k+n-i+\sum_{j=i}^{n-1} m_{j}>0
$$

and

$$
a_{i}(r)=\prod_{\substack{j=1 \\ j \neq i}}^{r} \frac{1}{\left(\gamma_{j}-\gamma_{i}\right)}, \quad 1 \leq i \leq r \leq n
$$


Theorem 2.1: For the Marshall-Olkin extended uniform distribution as given in (1.2) and $n \in$ $N, \tilde{m} \in \mathbb{R}, k>0,1 \leq r \leq n, p=1,2, \ldots$

$$
\begin{gathered}
E\left[X^{p+2}(r, n, \tilde{m}, k)\right]=\frac{\alpha \theta^{2}}{(1-\alpha)} E\left[X^{p}(r, n, \tilde{m}, k]+\frac{\theta \gamma_{r}}{(1-\alpha)(p+1)} E\left[X^{p+1}(r-1, n, \tilde{m}, k)\right]\right. \\
-\frac{\theta\left[\gamma_{r}-(p+1)(1-2 \alpha)\right]}{(1-\alpha)(p+1)} E\left[X^{p+1}(r, n, \tilde{m}, k)\right]
\end{gathered}
$$

Proof: We have by Athar and Islam (2004),

$$
\begin{aligned}
E[\xi\{X(r, n, \tilde{m}, k)\}] & -E[\xi\{X(r-1, n, \tilde{m}, k)\}] \\
& =C_{r-2} \int_{-\infty}^{\infty} \xi^{\prime}(x) \sum_{i=1}^{r} a_{i}(r)[\bar{F}(x)]^{\gamma_{i}} d x .
\end{aligned}
$$

Let $\xi(x)=x^{p+1}$, then

$$
\begin{aligned}
E\left[X^{p+1}(r, n, \tilde{m}, k)\right] & -E\left[X^{p+1}(r-1, n, \tilde{m}, k)\right] \\
= & C_{r-2}(p+1) \int_{-\infty}^{\infty} x^{p} \sum_{i=1}^{r} a_{i}(r)[\bar{F}(x)]^{\gamma_{i}} d x .
\end{aligned}
$$

Now in view of (1.4), we have

$$
\begin{aligned}
E & {\left[X^{p+1}(r, n, \tilde{m}, k)\right]-E\left[X^{p+1}(r-1, n, \tilde{m}, k)\right] } \\
& =\frac{(p+1)}{\theta \gamma_{r}} C_{r-1} \int_{0}^{\theta}(\theta-x)[\alpha \theta+(1-\alpha) x] x^{p} \sum_{i=1}^{r} a_{i}(r)[\bar{F}(x)]^{\gamma_{i}-1} f(x) d x .
\end{aligned}
$$

After simplification, we get the result.

Case II: $m_{i}=m, i=1,2, \ldots, n-1$.

The $p d f$ of $X(r, n, m, k)$ is given as

$$
f_{X(r, n, m, k)}(x)=\frac{C_{r-1}}{(r-1) !}[\bar{F}(x)]^{\gamma_{r}-1} f(x) g_{m}^{r-1}(F(x)),
$$

where,

$$
\begin{aligned}
& C_{r-1}=\prod_{i=1}^{r} \gamma_{i}, \gamma_{i}=k+(n-i)(m+1) \\
& h_{m}(x)= \begin{cases}-\frac{1}{m+1}(1-x)^{m+1} & , m \neq-1 \\
-\log (1-x) & , m=-1\end{cases}
\end{aligned}
$$


Haseeb Athar and Nayabuddin

and $\quad g_{m}(x)=h_{m}(x)-h_{m}(0), \quad x \in(0,1)$.

Theorem 2.2: For distribution as given in (1.2) and $n \in N, \tilde{m} \in \mathbb{R}, k>0,1 \leq r \leq n, p=1,2, \ldots$

$$
\begin{gathered}
E\left[X^{p+2}(r, n, m, k)\right]=\frac{\alpha \theta^{2}}{(1-\alpha)} E\left[X^{p}(r, n, m, k)\right]+\frac{\theta \gamma_{r}}{(1-\alpha)(p+1)} E\left[X^{p+1}(r-1, n, m, k)\right] \\
-\frac{\theta\left[\gamma_{r}-(p+1)(1-2 \alpha)\right]}{(1-\alpha)(p+1)} E\left[X^{p+1}(r, n, m, k)\right]
\end{gathered}
$$

Proof: It may be noted that for $\gamma_{i} \neq \gamma_{j}$ but at $m_{i}=m ; i=1,2, \ldots, n-1$,

$$
a_{i}(r)=\frac{1}{(m+1)^{r-1}}(-1)^{r-i} \frac{1}{(i-1) !(r-i) !}
$$

Therefore the $p d f$ of $X(r, n, \tilde{m}, k)$ given in (2.1) reduces to (2.3) [cf Khan et al., 2006].

Hence it can be seen that (2.4) is the partial case of (2.2) and is obtained by replacing $\tilde{m}$ with $m$ in (2.2).

Remark 2.1: Recurrence relation for single moments of order statistics (at $m=0, k=1$ ) is

$$
\begin{aligned}
E\left(X_{r: n}^{p+2}\right)= & \frac{\alpha \theta^{2}}{(1-\alpha)} E\left(X_{r: n}^{p}\right)+\frac{\theta(n-r+1)}{(1-\alpha)(p+1)} E\left(X_{r-1: n}^{p+1}\right) \\
& -\frac{\theta[(n-r+1)-(p+1)(1-2 \alpha)]}{(1-\alpha)(p+1)} E\left(X_{r: n}^{p+1}\right)
\end{aligned}
$$

Remark 2.2: Recurrence relation for single moments of $k-t$ th upper record (at $m=-1$ ) will be

$$
\begin{gathered}
E\left(X_{u(r)}^{(k)}\right)^{p+2}=\frac{\alpha \theta^{2}}{(1-\alpha)} E\left(X_{u(r)}^{(k)}\right)^{p}+\frac{\theta k}{(1-\alpha)(p+1)} E\left(X_{u(r-1)}^{(k)}\right)^{p+1} \\
-\frac{\theta[k-(p+1)(1-2 \alpha)]}{(1-\alpha)(p+1)} E\left(X_{u(r)}^{(k)}\right)^{p+1}
\end{gathered}
$$

and at $k=1$, we get the relation for the upper records, as obtained by Jose and Krishna (2011).

\section{Product Moments}

Case I: $\gamma_{i} \neq \gamma_{j} ; i, j=1,2, \ldots, n-1$

The joint probability density function ( $p d f)$ of $X(r, n, \tilde{m}, k)$ and $X(s, n, \tilde{m}, k), 1 \leq r<s \leq n$ is given as 


$$
\begin{aligned}
& f_{X(r, n, \tilde{m}, k), X(s, n, \tilde{m}, k)}(x, y)=C_{s-1}\left(\sum_{i=r+1}^{s} a_{i}^{(r)}(s)\left[\frac{\bar{F}(y)}{\bar{F}(x)}\right]^{\gamma_{i}}\right) \\
& \times\left(\sum_{i=1}^{r} a_{i}(r)[\bar{F}(x)]^{\gamma_{i}}\right) \frac{f(x)}{\bar{F}(x)} \frac{f(y)}{\bar{F}(y)}, \quad-\infty \leq x<y \leq \infty,
\end{aligned}
$$

where

$$
a_{i}^{(r)}(s)=\prod_{\substack{j=r+1 \\ j \neq i}}^{s} \frac{1}{\gamma_{j}-\gamma_{i}}, \quad r+1 \leq i \leq s \leq n .
$$

Theorem 3.1: For distribution as given in (1.2). Fix a positive integer $k$ and for $n \in N, \tilde{m} \in \mathbb{R}, 1 \leq$ $r<s \leq n$,

$$
\begin{gathered}
E\left[X^{p}(r, n, \tilde{m}, k) \cdot X^{q+2}(s, n, \tilde{m}, k)\right]=\frac{\alpha \theta^{2}}{(1-\alpha)} E\left[X^{p}(r, n, \tilde{m}, k) \cdot X^{q}(s, n, \tilde{m}, k)\right] \\
+\frac{\theta \gamma_{s}}{(1-\alpha)(q+1)} E\left[X^{p}(r, n, \tilde{m}, k) \cdot X^{q+1}(s-1, n, \tilde{m}, k)\right] \\
-\frac{\theta\left[\gamma_{s}-(q+1)(1-2 \alpha)\right.}{(1-\alpha)(q+1)} E\left[X^{p}(r, n, \tilde{m}, k) \cdot X^{q+1}(s, n, \tilde{m}, k)\right]
\end{gathered}
$$

Proof: We have by Athar and Islam (2004),

$$
\begin{gathered}
E[\xi\{X(r, n, \tilde{m}, k), X(s, n, \tilde{m}, k)\}]-E[\xi\{X(r, n, \tilde{m}, k), X(s-1, n, \tilde{m}, k)\}] \\
=C_{s-2} \iint_{-\infty \leq x<y \leq \infty} \frac{\partial}{\partial y} \xi(x, y) \sum_{i=r+1}^{s} a_{i}^{(r)}(s)\left[\frac{\bar{F}(y)}{\bar{F}(x)}\right]^{\gamma_{i}} \\
\times \sum_{i=1}^{r} a_{i}(r)[\bar{F}(x)]^{\gamma_{i}} \frac{f(x)}{\bar{F}(x)} d y d x .
\end{gathered}
$$

Now consider $\xi(x, y)=\xi_{1}(x) \cdot \xi_{2}(y)=x^{p} \cdot y^{q+1}$ in (3.3), then in view of (1.4) we get

$$
\begin{gathered}
E\left[X^{p}(r, n, \tilde{m}, k) \cdot X^{q+1}(s, n, \tilde{m}, k)\right]-E\left[X^{p}(r, n, \tilde{m}, k) \cdot X^{q+1}(s-1, n, \tilde{m}, k)\right] \\
=\frac{(q+1)}{\theta \gamma_{s}} C_{s-1} \int_{0}^{\theta} \int_{x}^{\theta}(\theta-y)[\alpha \theta+(1-\alpha) y] x^{p} y^{q} \sum_{i=r+1}^{s} a_{i}^{(r)}(s)\left[\frac{\bar{F}(y)}{\bar{F}(x)}\right]^{\gamma_{i}} \\
\times \sum_{i=1}^{r} a_{i}(r)[\bar{F}(x)]^{\gamma_{i}} \frac{f(x)}{\bar{F}(x)} \frac{f(y)}{\bar{F}(y)} d y d x
\end{gathered}
$$

which leads to (3.2). 
Haseeb Athar and Nayabuddin

Case II: $m_{i}=m ; i=1,2, \ldots, n-1$.

The joint $p d f$ of $X(r, n, m, k)$ and $X(s, n, m, k), 1 \leq r<s \leq n$ is given as

$$
\begin{gathered}
f_{X(r, n, m, k), X(s, n, m, k)}(x, y)=\frac{C_{s-1}}{(r-1) !(s-r-1) !}[\bar{F}(x)]^{m} f(x) g_{m}^{r-1}(F(x)) \\
\times\left[h_{m}(F(y))-h_{m}(F(x))\right]^{s-r-1}[\bar{F}(y)]^{\gamma_{s}-1} f(y),-\infty \leq x<y \leq \infty .
\end{gathered}
$$

Theorem 3.2: For distribution as given in (1.2) and condition stated as in Theorem 3.1

$$
\begin{gathered}
E\left[X^{p}(r, n, m, k) \cdot X^{q+2}(s, n, m, k)\right]=\frac{\alpha \theta^{2}}{(1-\alpha)} E\left[X^{p}(r, n, m, k) \cdot X^{q}(s, n, m, k)\right] \\
+\frac{\theta \gamma_{s}}{(1-\alpha)(q+1)} E\left[X^{p}(r, n, m, k) \cdot X^{q+1}(s-1, n, m, k)\right] \\
-\frac{\theta\left[\gamma_{s}-(q+1)(1-2 \alpha)\right.}{(1-\alpha)(q+1)} E\left[X^{p}(r, n, m, k) \cdot X^{q+1}(s, n, m, k)\right]
\end{gathered}
$$

Proof: We have when $\gamma_{i} \neq \gamma_{j}$ but at $m_{i}=m, i=1,2, \ldots, n-1$

$$
a_{i}^{(r)}(s)=\frac{1}{(m+1)^{s-r-1}}(-1)^{s-i} \frac{1}{(i-r-1) !(s-i) !}
$$

Hence, joint $p d f$ of $X(r, n, \tilde{m}, k)$ and $X(s, n, \tilde{m}, k)$ given in (3.1) reduces to (3.4). [cf Khan et al., 2006].

Therefore, Theorem 3.2 can be established by replacing $\tilde{m}$ with $m$ in Theorem 3.1 .

Remark 3.1: Recurrence relation for product moments of order statistics (at $m=0, k=1$ ) is

$$
\begin{aligned}
E\left(X_{r, s: n}^{p, q+2}\right)= & \frac{\alpha \theta^{2}}{(1-\alpha)} E\left(X_{r, s: n}^{p, q}\right)+\frac{\theta(n-s+1)}{(1-\alpha)(q+1)} E\left(X_{r, s-1: n}^{p, q+1}\right) \\
& \frac{\theta[(n-s+1)-(q+1)(1-2 \alpha)]}{(1-\alpha)(q+1)} E\left(X_{r, s: n}^{p, q+1}\right)
\end{aligned}
$$

Remark 3.2: Recurrence relation for product moments of $k-t h$ record values will be

$$
\begin{aligned}
E\left[\left(X_{u(r)}^{(k)}\right)^{p} \cdot\left(X_{u(s)}^{(k)}\right)^{q+2}\right] & =\frac{\alpha \theta^{2}}{(1-\alpha)} E\left[\left(X_{u(r)}^{(k)}\right)^{p} \cdot\left(X_{u(s)}^{(k)}\right)^{q}\right] \\
& +\frac{\theta k}{(1-\alpha)(q+1)} E\left[\left(X_{u(r)}^{(k)}\right)^{p} \cdot\left(X_{u(s-1)}^{(k)}\right)^{q+1}\right]
\end{aligned}
$$




$$
-\frac{\theta[k-(p+1)(1-2 \alpha)]}{(1-\alpha)(q+1)} E\left[\left(X_{u(r)}^{(k)}\right)^{p} \cdot\left(X_{u(s)}^{(k)}\right)^{q+1}\right]
$$

Remark 3.3: At $p=0$, we obtain recurrence relation for single moments as given in (2.2) and (2.4).

\section{Characterization}

Theorem 4.1: Let $X$ be an absolutely continuous random variable with $c d f F(x)$ and $p d f f(x)$. Suppose $0<F(x)<1$ for all $x>0$, then

$$
\begin{array}{r}
E\left[X^{p+1}(r, n, m, k)\right]-E\left[X^{p+1}(r-1, n, m, k)\right] \\
=\frac{(p+1)}{\theta \gamma_{r}}\left[\alpha \theta^{2} E\left[X^{p}(r, n, m, k)\right]+\theta(1-2 \alpha) E\left[X^{p+1}(r, n, m, k)\right]\right. \\
\left.-(1-\alpha) E\left[X^{p+2}(r, n, m, k)\right]\right]
\end{array}
$$

if and only if

$$
\bar{F}(x)=\frac{\alpha(\theta-x)}{[\alpha \theta+(1-\alpha) x]}, 0<x<\theta, \quad \alpha>0 .
$$

where, $\bar{F}(x)=1-F(x)$.

Proof: Necessary part follows from Theorem 2.2.

To proof (4.1) implies (4.2), we have

$$
\begin{aligned}
& \alpha \theta^{2} \frac{C_{r-1}}{(r-1) !} \int_{0}^{\theta} x^{p}[\bar{F}(x)]^{\gamma_{r}-1} g_{m}^{r-1}(F(x)) f(x) d x \\
& +(1-2 \alpha) \theta \frac{C_{r-1}}{(r-1) !} \int_{0}^{\theta} x^{p+1}[\bar{F}(x)]^{\gamma_{r}-1} g_{m}^{r-1}(F(x)) f(x) d x \\
& -(1-\alpha) \frac{C_{r-1}}{(r-1) !} \int_{0}^{\theta} x^{p+2}[\bar{F}(x)]^{\gamma_{r}-1} g_{m}^{r-1}(F(x)) f(x) d x \\
& -\theta \frac{C_{r-1}}{(r-1) !} \int_{0}^{\theta} x^{p}[\bar{F}(x)]^{\gamma_{r}} g_{m}^{r-1}(F(x)) d x=0
\end{aligned}
$$


which may be re-written as

$$
\frac{C_{r-1}}{(r-1) !} \int_{0}^{\theta} x^{p}[\bar{F}(x)]^{\gamma_{r}-1} g_{m}^{r-1}(F(x)) f(x)\left[(\theta-x)[\alpha \theta+(1-\alpha) x]-\theta \frac{\bar{F}(x)}{f(x)}\right] d x=0 .
$$

Now applying a generalization of Müntz - Szász Theorem (Hwang and Lin, 1984), we get

$$
\frac{f(x)}{\bar{F}(x)}=\frac{\theta}{(\theta-x)[\alpha \theta+(1-\alpha) x]}
$$

and hence the (4.2).

\section{Acknowledgement}

Authors are thankful to Professor M. Ahsanullah, Editor in Chief, JSTA and learned referee who spent their valuable time to review this manuscript.

\section{References}

[1] Athar, Haseeb and Islam, H.M. (2004): Recurrence relations between single and product moments of generalized order statistics from a general class of distributions. Metron, LXII, 327-337.

[2] Hwang, J.S. and Lin, G.D. (1984): On a generalized moments problem II. Proc. Amer. Math. Soc., 91, $577-580$.

[3] Jose, K.K. and Krishna, E. (2011): Marshall-Olkin Extended Uniform Distribution. ProbStat Forum, 4, $78-88$.

[4] Kamps, U. (1995): A concept of generalized order statistics. B.G. Teubner Stuttgart, Germany.

[5] Khan, A. H., Khan, R. U. and Yaqub, M. (2006): Characterization of continuous distributions through conditional expectation of generalized order statistics. J. Appl. Prob. Statist., 1, 115-131. 